Original Article

\title{
Solid-state characterization studies and effect of PEG 20000 and P90G on particle size reduction and stability of complexed glimepiride nanocrystals
}

\author{
Babasahib Sajeev Kumar ${ }^{\mathrm{a}, *, \mathrm{~d}}$, Raman Saraswathi ${ }^{\mathrm{b}}$, Sokkalingam Arumugham Dhanaraj ${ }^{\mathrm{c}}$ \\ a Department of Pharmaceutics, Jawaharlal Nehru Technological University, Hyderabad, Andra Pradesh 500 085, India \\ ${ }^{\mathrm{b}}$ Department of Pharmaceutics, National College of Pharmacy, Kozhikode 673 602, Kerala, India \\ ${ }^{\mathrm{c}}$ Faculty of Pharmacy, Asian Institute of Medicine Science and Technology, 08100 Bedong, Malaysia
}

\section{A R T I C L E I N F O}

Article history:

Received 3 July 2013

Accepted 11 August 2013

Available online 26 September 2013

\section{Keywords:}

Lipid nanocrystals

Precipitation

Surface properties

Crystallinity

Nanonization

\begin{abstract}
A B S T R A C T
Objective: The objective of the present study is to formulate and characterize the properties of complexed glimepiride nanocrystals (GLP) by various techniques at different stages of its development, and to study the effect of PEG 20000 and P90G on particle size reduction and stability of nanocrystals. Method: Precipitated (GLP-PEG) and complexed NCs (GLP-PEG-P90G) of glimepiride were characterized for particle size, size distribution, zeta potential and stability assessment using photon correlation spectroscopy (PCS). The crystallinity was analyzed using differential scanning calorimetry (DSC) and Xray powder diffraction spectroscopy (XRPD). The surface morphology and chemical stability were assessed by means of scanning electron microscopy (SEM) and infrared spectroscopy (FTIR).

Results: A formulation with drug-polymer ratio of $1: 1$ was most ideal in developing stable NCs as it exhibited smaller particle size and high stability. A high zeta potential was observed in all NCs after complexation indicating improved stability. DSC and XRPD studies showed no change in crystallinity after complexation. SEM analysis of complexed NCs showed presence of spherical shape particles (size below $1 \mu \mathrm{m}$ ) with a lipid coat on the surface. Stability studies on optimized formulation (F1) revealed no change in particle size during 3-month period. FTIR studies prove that the chemical identity of GLP was preserved in the samples and the formulation was stable.

Conclusion: Solid-state characterization studies reveal that complexed GLP NCs are promising carriers for drug delivery and they can be safely and effectively used in design of various formulations. Also, PEG 20000 and P90G are excellent polymer and lipid for particle size reduction (nanonization) and stabilization of nanocrystals.
\end{abstract}

Copyright @ 2013, InPharm Association, Published by Reed Elsevier India Pvt. Ltd. All rights reserved.

\section{Introduction}

Solid-state characterization studies are widely used to explore the comprehensive structure-functional relationships between the nanoparticle structure and pharmacological properties of a drug. This could help in uncomplicated development, easy adoption and safe administration of a drug., ${ }^{1,2}$ Currently different nanoparticulate systems are in progressive research to improve the bioavailability of a drug. ${ }^{3}$ The performance of these carriers depends on variety of factors like the surface properties, particle size, shape, composition and its stability both in vitro and in vivo. ${ }^{4}$

\footnotetext{
* Corresponding author. Tel.: +916016 456 3044; fax: +9160604 4298009. E-mail address: bsajeev2001@yahoo.com (B. Sajeev Kumar).

d B. SK has contributed in design and development of the formulation.
}

The above factors have to be fully established and their effects on drug pharmacokinetics and pharmacodynamics need to be clearly elucidated to assess the safety and competence of various nanoformulations. ${ }^{5}$

Compared to all nanoparticulates, nanocrystals (NCs) are considered to be the least complex, as it contain 100\% drug with no carriers. ${ }^{6}$ Due to their nanosize, they offer excellent solubility and are considered to solve the issues of poor solubility. ${ }^{7,8} \mathrm{NCs}$ possess major limitations like crystal growth (aggregation) on contact with fluids or electrolytes, and loss in its functional properties. ${ }^{9} \mathrm{~A}$ strategy to overcome the limitations is to alter the surface properties of NCs by attaching ligands to them or by increasing its stealthiness. ${ }^{10}$ This approach could decrease particle aggregation and improve in vivo stability. ${ }^{11}$

During the production process, real time monitoring of immediate NCs and assurance test of final product are necessary. This is 
Table 1

Particle characterization data of GLP NCs.

\begin{tabular}{|c|c|c|c|c|c|c|c|}
\hline \multirow[t]{2}{*}{ Batch } & \multirow[t]{2}{*}{ Drug:polymer } & \multicolumn{3}{|c|}{ Precipitated NCs } & \multicolumn{3}{|c|}{ Complexed NCs } \\
\hline & & Z.avg (d nm) & PDI (avg.) & Avg. ZP (mV) & Z.avg (d nm) & PDI (avg.) & Avg. ZP (mV) \\
\hline Pure GLP & $1: 0$ & $2066 \pm 0.351$ & $0.252 \pm 0.25$ & $-40.2 \pm 0.01$ & - & - & - \\
\hline $\mathrm{F} 1$ & $1: 1$ & $1650 \pm 0.017$ & $0.367 \pm 0.13$ & $-40.7 \pm 0.01$ & $240 \pm 0.027$ & $0.766 \pm 0.15$ & $-59.0 \pm 0.05$ \\
\hline F2 & $1: 2$ & $1920 \pm 0.042$ & $0.460 \pm 0.01$ & $-42.1 \pm 0.12$ & $550 \pm 0.162$ & $0.604 \pm 0.04$ & $-54.4 \pm 0.03$ \\
\hline F3 & $1: 4$ & $1593 \pm 0.138$ & $0.673 \pm 0.04$ & $-41.4 \pm 0.04$ & $281 \pm 0.055$ & $1.0 \pm 0.07$ & $-51.7 \pm 0.16$ \\
\hline $\mathrm{F} 4$ & $1: 8$ & $307 \pm 0.252$ & $0.743 \pm 0.06$ & $-39.1 \pm 0.21$ & $210 \pm 0.039$ & $0.847 \pm 0.18$ & $-51.5 \pm 0.21$ \\
\hline F5 & $2: 1$ & $30 \pm 0.041$ & $0.212 \pm 0.32$ & $-31.6 \pm 0.47$ & $249 \pm 0.185$ & $1.0 \pm 0.06$ & $-41.7 \pm 0.04$ \\
\hline
\end{tabular}

\pm Indicates SD $(n=3)$.
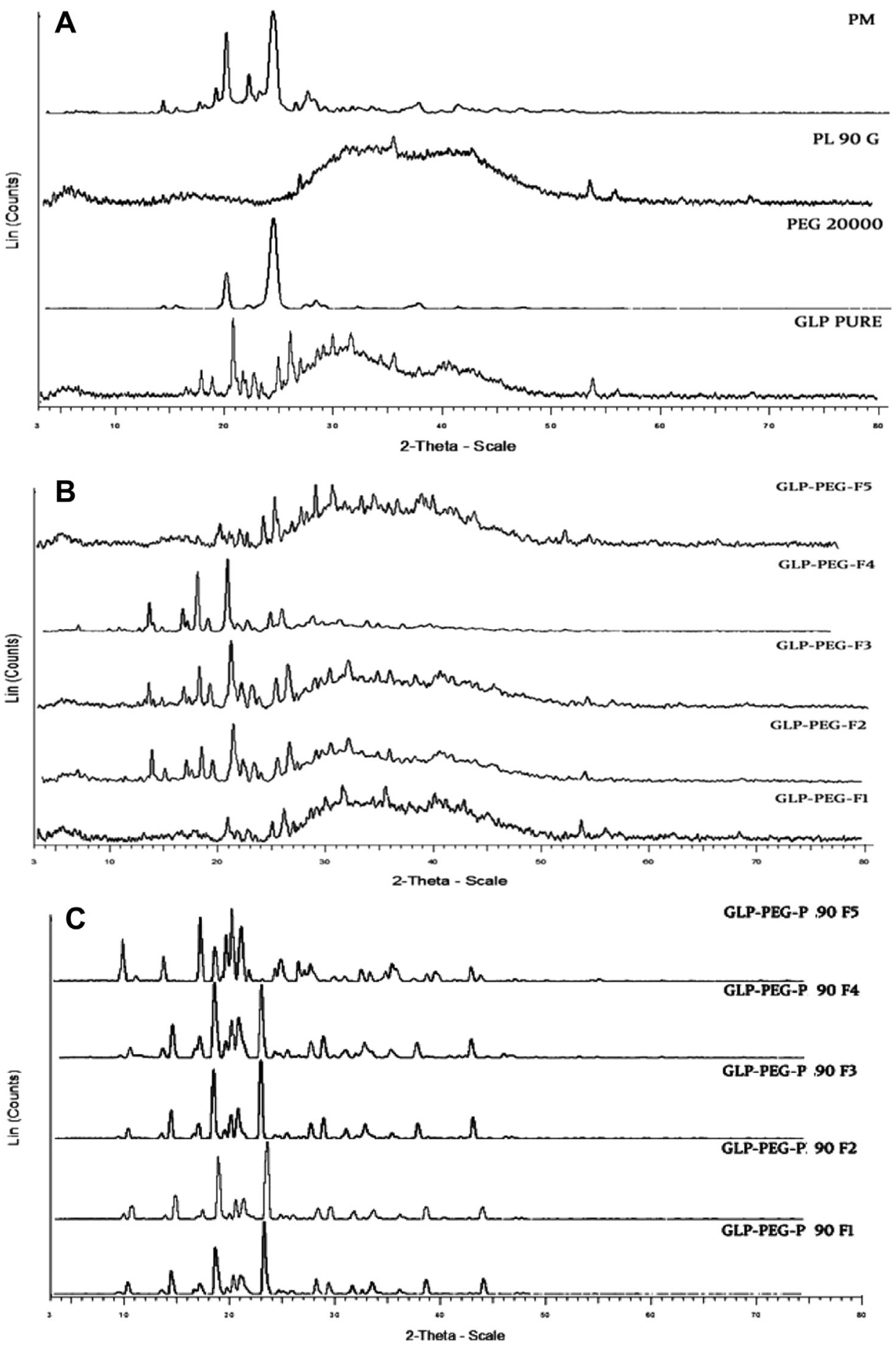

Fig. 1. XRPD of pure GLP, PEG 20000, PL90G and PM (1:1) (A), precipitated GLP NCs (F1-F5) (B) and complexed GLP NCs (C) at 2-Theta-scale. 
critical in product development as it assist in evaluating the performance of the NCs in drug delivery. ${ }^{12}$ Parameters like particle size, surface charge, size distribution, crystallinity and aggregation need to be controlled precisely as they may affect the ADME (absorption, distribution, metabolism and excretion) and toxicity of nanoformulation. ${ }^{13}$

The objective of the present study is to characterize the properties of Glimepiride NCs (GLP) by various techniques at different stages of its formulation. NCs were developed by precipitation process using PEG 20000 and stabilized (complexed) by means of Phospholipon 90G (P90G). Glimepiride is a weak acidic, second generation oral hypoglycemic agent (BCS Class II drug), with high permeability, low aqueous solubility $\left(\sim 3.84-02 \mathrm{mg} / \mathrm{ml}\right.$ at $\left.37{ }^{\circ} \mathrm{C}\right)$ and poor dissolution rate. ${ }^{14-16}$ It has a $\log P$ value of 3.2 and a $\mathrm{pKa}$ of 6.2. GLP is a drug of choice for long-term therapy for diabetes mellitus and it requires rapid GI absorption to prevent a sudden increase in the blood glucose level after food intake. ${ }^{17}$ Particle and solid-state characterization studies facilitate in development of a stable formulation with fewer drugs-excipient interactions and it enables to design a formulation with improved therapeutic efficiency.

\section{Materials and methods}

\subsection{Materials}

Glimepiride was obtained as a gift sample from S.D Biomed (Malaysia). PEG 20000 and P90G were procured from Sigma Aldrich and $\mathrm{GmbH}$, Germany. Acetone, Tween 80, sodium dodecyl sulfate, polysorbate 80 , dichloromethane and methanol were purchased from R \& M Chemicals, (Malaysia). Deionized water was obtained from Millipore, MilliQ-Plus. All the other solvents and reagents used were of Anala $R$ grade.

\subsection{Methods}

GLP was dissolved in dichloromethane (DCM). PEG 20000 was added to the drug solution and stirred (Erla-EMS H7000, Korea) at a temperature not exceeding $60{ }^{\circ} \mathrm{C}$. The drug-polymer solution was injected slowly $(1 \mathrm{ml} / \mathrm{min})$ into an aqueous phase containing Tween $80(2.5 \% \mathrm{w} / \mathrm{v})$ with mechanical stirring at $450 \mathrm{rpm}$ to precipitate the NCs. The volume $(80 \mathrm{ml})$ of dispersion was adjusted to $100 \mathrm{ml}$ using double distilled water and stirred for $4 \mathrm{~h}$ at room temperature. Later, the solution was gently heated $\left(65^{\circ} \mathrm{C}\right)$ with magnetic stirring for $45 \mathrm{~min}$ to remove the organic solvent. The contents were centrifuged (Heraeus-Labofuge 200, Germany) at $5000 \mathrm{rpm}$ for $20 \mathrm{~min}$ to separate the NCs. The clear supernatant liquid was discarded; the thick viscous dispersion was collected and further redispersed in $15 \mathrm{ml}$ of distilled water and recentrifuged $(20,000 \mathrm{rpm})$ for $10 \mathrm{~min}$ to remove the impurities and the residual surfactants. The precipitated NCs were recovered using a vacuum filter (Kontes Ultra ware $-0.2 \mu \mathrm{m}$, USA) and dried in a hot air oven (Memmert - UF110, Germany) at $35{ }^{\circ} \mathrm{C}$ for $20 \mathrm{~min}$. The procedure was repeated with different drug-carrier ratio to obtain various batches.

\subsection{Complexation of GLP-PEG NCS}

$50 \mathrm{mg}$ of dried NCs were accurately weighed and dispersed in $50 \mathrm{ml}$ of phosphate buffer ( $\mathrm{pH} \mathrm{7.8)}$ in presence of Tween $80(0.1 \%$ $\mathrm{w} / \mathrm{v})$ by gentle stirring for $10 \mathrm{~min}$. P90G $(2 \% \mathrm{w} / \mathrm{v})$ previously solubilized in chloroform was gradually added ( $3 \mathrm{ml} / \mathrm{min}$ ) to the dispersion and stirred using a magnetic stirrer at $250 \mathrm{rpm}$ for $30 \mathrm{~min}$ at a temperature above its melting point $\left(60^{\circ} \mathrm{C}\right)$. The dispersion was shaken (Daiki Scientific - DK-SI 010, Korea) at
$120 \mathrm{rpm}$ for $1 \mathrm{~h}$ at $15^{\circ} \mathrm{C}$. Mannitol (5\% w/v) was added to the dispersion and shaken for $10 \mathrm{~min}$ prior to lyophilization.

\subsection{Freeze drying}

The milky homogenous dispersion "prepared in 2.3 above" was subjected to freeze drying in a freeze dryer (Thermo scientific, USA), with an inbuilt Pirani 501 microprocessor. The samples were lyophilized at a slow freezing temperature (shelf temperature $-40^{\circ} \mathrm{C}$ at 6 torr and $10^{-1}$ mbar pressure) for $10 \mathrm{~h}$. The lyophilized products were stored in borosilicate glass vials and stored in a dessicator at room temperature until further use.

\subsection{Particle characterization}

\subsubsection{Photon correlation spectroscopy (PCS)}

The mean particle size and polydispersity index (PDI) were measured using Malvern Zetasizer Nano ZS (Malvern Instruments, UK). $2 \mathrm{mg}$ of sample was dispersed in $150 \mathrm{ml}$ of deionized water containing $0.25 \% \mathrm{w} / \mathrm{v}$ of Tween 80 and $0.5 \% \mathrm{w} / \mathrm{v}$ of sodium dodecyl sulfate (SDS). The dispersion was sonicated in a bath sonicator (Power sonic 410 , Lab Tech, Korea) and left aside for $24 \mathrm{~h}$ prior to analysis. $4 \mu \mathrm{l}$ of each suspension was diluted with $2 \mathrm{ml}$ of deionized water and the samples were pipetted into disposable polystyrene cuvette. The
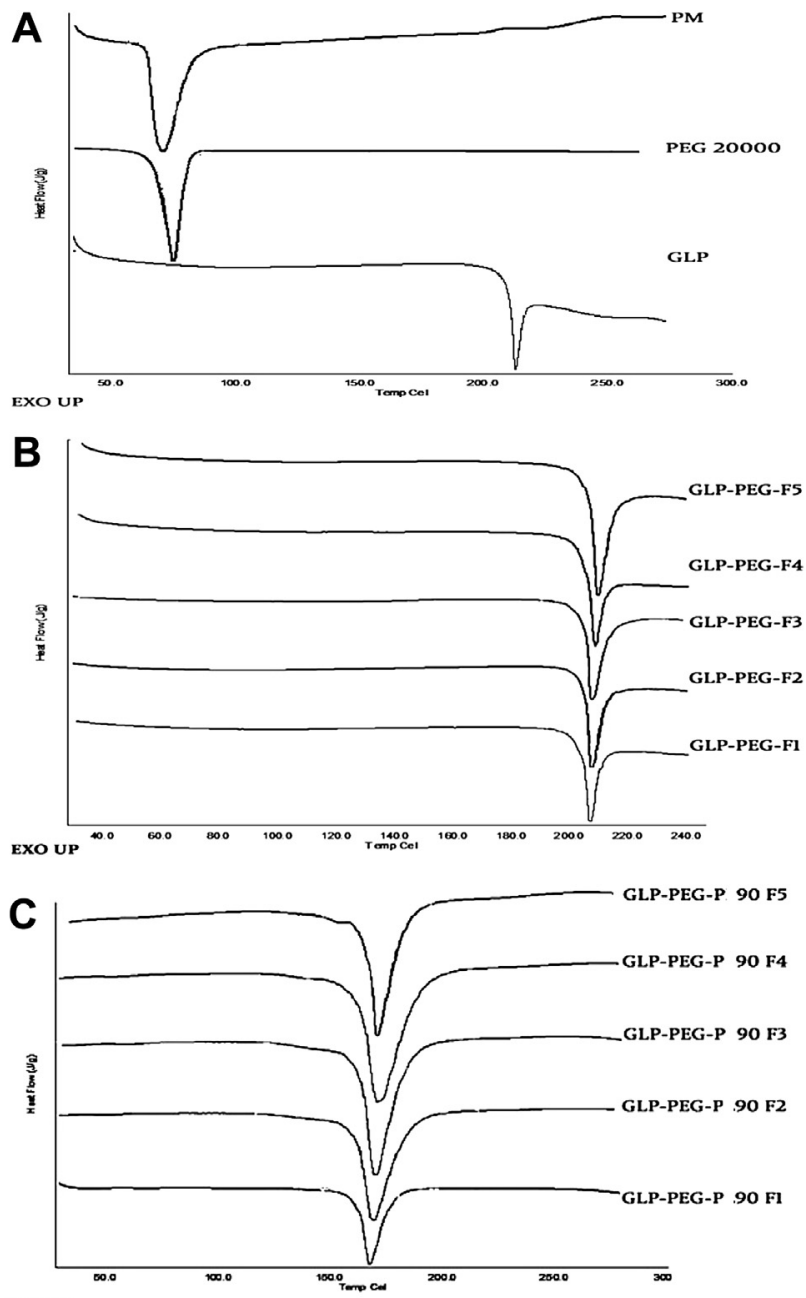

EXO UP

Fig. 2. DSC thermograms of pure GLP, PEG 20000 and PM (1:1) (A), precipitated GLP NCs (B) and complexed GLP NCS (C). 
samples were measured for mean particle size and PDI at a fixed angle of $90^{\circ}$ at a temperature of $25^{\circ} \mathrm{C}$ after 5 runs. A refractive index of 1.590 and 1.300 was used for the drug and solvent respectively.

\subsubsection{Zeta potential measurement (ZP)}

The zeta potential was measured using Malvern Zetasizer Nano ZS (M3-PALS, Malvern Instruments, UK). Samples were dispersed in deionized water and left for $24 \mathrm{~h}$, and were injected into a clear disposable zeta cell after suitable dilution. The zeta cell was checked for presence of air bubbles and if present was removed by tapping. The average zeta potential $(\mathrm{mV})$ was measured after 3 scans.

\subsection{Solid-state characterization}

\subsubsection{X-ray powder diffraction spectroscopy (XRPD)}

XRPD diffractograms of pure GLP, PEG 20000 physical mixtures (1:1) and NCs were recorded in X-ray diffractometer (Bruker AXS D8, Germany) with Anton Paar, TTK 450 temperature attachment, using Si (Li) PSD detector. The samples were placed in a glass sample holder and $\mathrm{Cu}$ ka radiation was generated at $30 \mathrm{~mA}$ and 40 $\mathrm{Kv}$. The samples were scanned from $3^{\circ}$ to $80^{\circ}$ with a step size of $0.02^{\circ}$.

\subsubsection{Differential scanning calorimetry (DSC)}

DSC analysis of pure GLP, PEG 20000, physical mixture of drug and polymer (1:1) and NCs were analyzed in a DSC calorimeter (TA Instruments, Q200, USA), equipped with a liquid nitrogen cooling system. About $5 \mathrm{mg}$ of samples were loaded into an aluminum pan, crimped, sealed and further examined at a scanning rate of $10{ }^{\circ} \mathrm{C} / \mathrm{min}$ from 15 to $200{ }^{\circ} \mathrm{C}$ under nitrogen atmosphere (flow rate $100 \mathrm{ml} / \mathrm{min}$ ) at room temperature. High purity indium was used to calibrate the heat flow and heat capacity of the instruments.

\subsubsection{FTIR analysis}

Spectra of pure GLP, PEG 20000, physical mixture (1:1) and NCs were recorded in FT-IR spectrophotometer (Thermo Nicolet, Avatar 370, USA). The samples were compressed into a pellet using $\mathrm{KBr}$
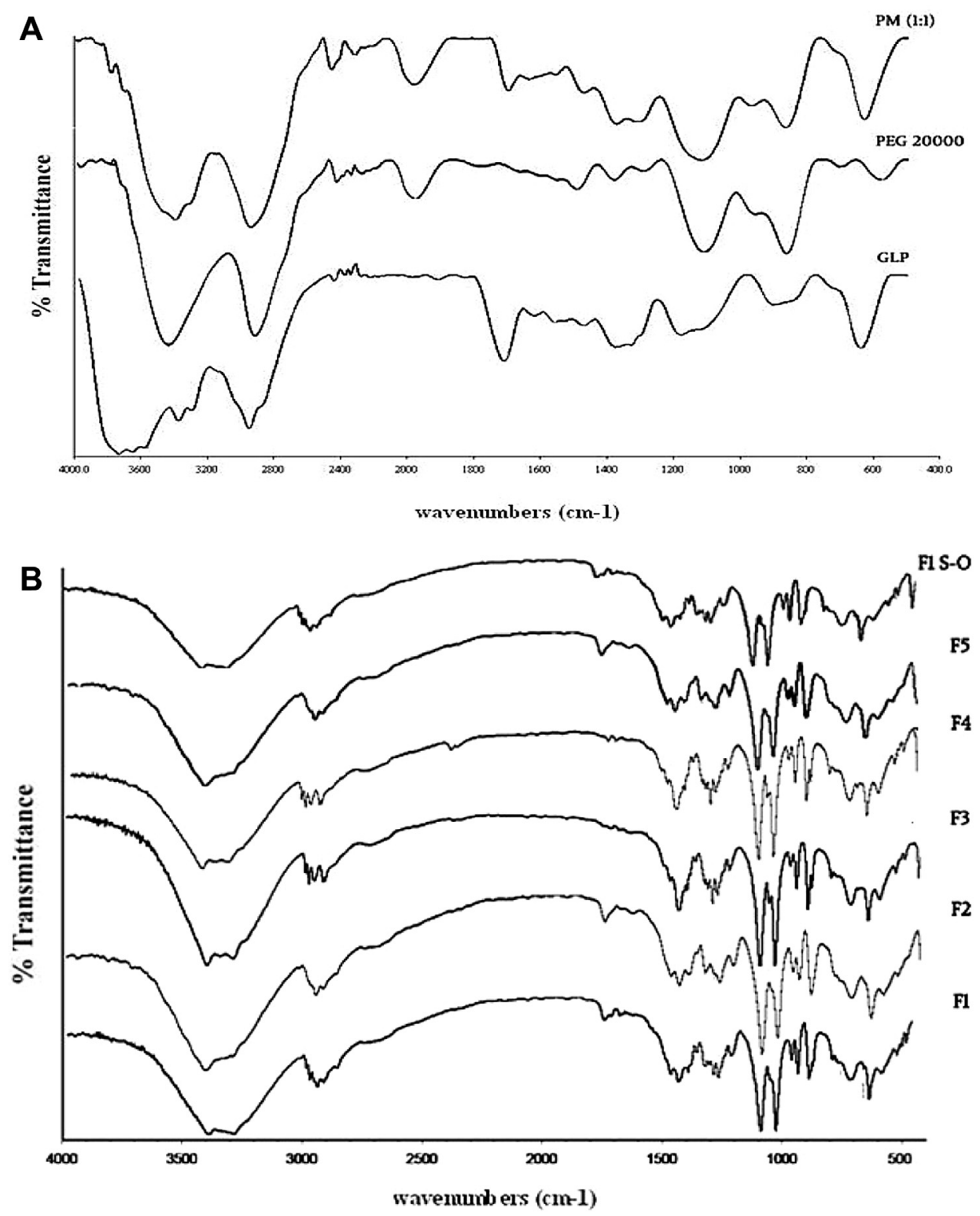

Fig. 3. FTIR spectra of pure GLP, PEG 20000 and PM (1:1) (A), precipitated GLP NCs (F1-F5) and optimized formulation (F1-S-O) after 3 months of storage (B). 
and scanned for $4 \mathrm{~s}$ at a resolution of $4 \mathrm{~cm}^{-1}$ from 4000 to $400 \mathrm{~cm}^{-1}$.

\subsubsection{Scanning electron microscopy analysis (SEM)}

Morphological evaluation of NCs was performed using a scanning electron microscope (LEO 1530, Gemini, Germany). The samples were mounted to steel stubs (Jeol $-10 \mathrm{~mm}$ Dia $\times 5 \mathrm{~mm}$ ) using a double sided adhesive tape and sputtered with a thin layer of $\mathrm{Au}$ at $20 \mathrm{~mA}$, under $1 \times 10^{-1}$ bar vacuum for $10 \mathrm{~min}$ using a sputter coater (EM S550X - Electron microscopy sciences) and was operated at an acceleration voltage of $3 \mathrm{kV}$.

\subsection{Stability studies}

The optimized formulation (Batch F1) was placed in a clean airtight glass vials and stored at different temperature conditions (room temperature and $40{ }^{\circ} \mathrm{C} / 75 \% \mathrm{RH}$ ) over a period of 3 months. During the storage period, the samples were subjected to particle size measurement and observed for any shift in FT-IR spectra.

\section{Results}

\subsection{Photon correlation spectroscopy}

\subsubsection{Effect of polymer on particle size reduction}

The particle size analysis data of precipitated and complexed NCs are shown in Table 1 . The particle size was found to vary with polymer concentration in precipitated NCs. The average particle size of pure GLP was found to be $2066 \mathrm{~nm}$, while that of precipitated NCs (F1-F5) were between 30 and $1920 \mathrm{~nm}$. The mean particle size of complexed NCs was in the range of $210-550 \mathrm{~nm}$ and was found to decrease after complexation. The PDI of precipitated and complexed NCs were found to be higher in comparison to pure GLP (0.252) and possessed a broader distribution.

\subsubsection{Effect of zeta potential and stability of NCS}

The average zeta potential of pure GLP, precipitated and complexed NCs are compared in Table 1. The zeta potential of pure GLP was found to be $-40.2 \mathrm{mV}$, while that of precipitated NCs were between -31.6 and $-42.1 \mathrm{mV}$. The zeta potential was found to increase $(>-40 \mathrm{mV})$ in all batches after complexation signifying an increase in stability as compared to pure GLP.

\subsection{X-ray powder diffraction spectroscopy}

The diffraction spectra of pure GLP, PEG 20000, PM (1:1), precipitated and complexed NCs are illustrated in Fig. $1 \mathrm{~A}-\mathrm{C}$ respectively. Numerous sharp and narrow intense peaks were observed at $17.82^{\circ}, 20.77^{\circ}, 21.06^{\circ}, 22.68^{\circ}, 24.91^{\circ}, 26.02^{\circ}$ and $53.82^{\circ}$ in pure GLP spectra proving its high crystallinity. The XRPD spectra of PM showed less intense peaks with decrease in peak area. Numerous low intense, slightly broadened peaks with reduced sharpness and low peak area were observed in the spectra of all NCs. These spectral changes may be attributed to the changes in crystal size of samples. XRPD peak parameters of GLP, precipitated and complexed NCs are given in Table 2.

\subsection{Differential scanning calorimetry}

DSC thermograms of GLP, PEG 20000, PM (1:1), precipitated and complexed NCs are compared in Fig. $2 \mathrm{~A}-\mathrm{C}$ respectively. A sharp endothermic peak at $213.8{ }^{\circ} \mathrm{C}(\Delta H=43.1 \mathrm{~J} / \mathrm{g})$ in the pure GLP thermogram indicated its high crystallinity. An endothermic peak at $65.24{ }^{\circ} \mathrm{C}$ in PEG 20000 thermogram revealed it is crystalline in nature $(\Delta H=187.6 \mathrm{~J} / \mathrm{g})$. A single endothermic peak at $69.9{ }^{\circ} \mathrm{C}$
Table 2

XRPD peak parameters of GLP and formulations.

\begin{tabular}{|c|c|c|c|c|c|c|}
\hline \multirow[t]{2}{*}{ Batch } & \multicolumn{3}{|c|}{ Precipitated NCs } & \multicolumn{3}{|c|}{ Complexed NCs } \\
\hline & $\begin{array}{l}2 \theta \\
\text { position }\end{array}$ & $\begin{array}{l}\text { Peak intensity } \\
\text { (d) }\end{array}$ & $\begin{array}{l}\text { FWHM } \\
\text { (deg) }\end{array}$ & $\begin{array}{l}2 \theta \\
\text { position }\end{array}$ & $\begin{array}{l}\text { Peak intensity } \\
\text { (d) }\end{array}$ & $\begin{array}{l}\text { FWHM } \\
\text { (deg) }\end{array}$ \\
\hline Pure & 17.82 & 4.97 & - & - & - & - \\
\hline \multirow[t]{5}{*}{ GLP } & 20.77 & 4.27 & - & - & - & - \\
\hline & 22.68 & 3.91 & - & - & - & - \\
\hline & 24.91 & 3.57 & - & - & - & - \\
\hline & 26.02 & 3.42 & - & - & - & - \\
\hline & 53.82 & 1.70 & - & - & - & - \\
\hline \multirow[t]{5}{*}{ F1 } & 20.77 & 4.27 & - & 18.92 & 4.66 & 0.42 \\
\hline & 22.64 & 3.92 & - & - & - & - \\
\hline & 24.91 & 3.57 & - & 23.55 & 3.77 & 3.31 \\
\hline & 26.00 & 3.42 & - & 28.43 & 3.13 & - \\
\hline & 53.73 & 1.70 & - & - & - & - \\
\hline \multirow[t]{4}{*}{ F2 } & 18.02 & 4.91 & - & 18.90 & 4.66 & 0.36 \\
\hline & 20.92 & 4.23 & - & - & - & - \\
\hline & 25.11 & 3.54 & - & 23.66 & 3.75 & 0.43 \\
\hline & 26.23 & 3.39 & - & - & - & - \\
\hline \multirow[t]{6}{*}{ F3 } & 17.92 & 4.94 & - & - & - & - \\
\hline & 18.92 & 4.63 & - & 19.01 & 4.66 & 0.34 \\
\hline & 20.80 & 4.25 & - & 20.70 & 4.41 & 0.32 \\
\hline & 22.78 & 3.90 & - & 23.63 & 3.76 & 0.34 \\
\hline & 25.0 & 3.5 & - & - & - & - \\
\hline & 26.10 & 3.41 & - & - & - & - \\
\hline \multirow[t]{5}{*}{ F4 } & 18.04 & 4.91 & 0.28 & 19.06 & 4.65 & 0.43 \\
\hline & 20.97 & 4.23 & 0.33 & - & - & - \\
\hline & 22.89 & 3.88 & - & 23.67 & 3.75 & 0.38 \\
\hline & 26.24 & 3.39 & 0.30 & - & - & - \\
\hline & 22.51 & 3.93 & - & - & - & - \\
\hline \multirow[t]{2}{*}{ F5 } & 24.84 & 3.58 & - & 21.60 & 11.24 & 0.41 \\
\hline & 25.94 & 3.43 & - & 24.41 & 2.67 & 0.38 \\
\hline
\end{tabular}

FWHM - full width half maximum.

( $\Delta H=71.0 \mathrm{~J} / \mathrm{g}$ ) were observed in the thermogram of physical mixture (1:1), due to the fusion of the components and indicated some modifications due to the presence of PEG 20000.

The endothermic peak values of all precipitated NCs were between 212.6 and $213.3^{\circ} \mathrm{C}$, and were comparable to the peak values of pure GLP. The $\Delta H(\mathrm{~J} / \mathrm{g})$ of NCs was found to slightly vary within the batches. Incase of complexed NCs, the endothermic peak values were found to be lower and were between 166.7 and $167.7{ }^{\circ} \mathrm{C}$.

\subsection{FT-IR analysis}

FT-IR spectra of pure GLP, PEG 20000, PM (1:1) and precipitated NCs are compared in Fig. 3A and B respectively. Pure GLP exhibited characteristic bands at $3366 \mathrm{~cm}^{-1}$ and $2946 \mathrm{~cm}^{-1}(\mathrm{NH}$ and $\mathrm{C}-\mathrm{H}$ aromatic stretching), $1683 \mathrm{~cm}^{-1}$ (methylene cyclohexane), $1532 \mathrm{~cm}^{-1}$ and $1443 \mathrm{~cm}^{-1}\left(\mathrm{~N}=\mathrm{O}\right.$ stretching), $1146 \mathrm{~cm}^{-1}$ and $1081 \mathrm{~cm}^{-1}$ (C-O stretching and sulfoxide group respectively). ${ }^{18}$ The presence of characteristic peaks of GLP in all NC formulations proved the absence of interaction between drug and the polymer.

\subsection{Surface characteristic analysis}

The SEM images of pure GLP (Fig. 4A) showed numerous irregularly shaped particles of large size $(>5 \mu \mathrm{m})$. The precipitated NCs were polyhedron in shape and were aggregated before complexation (Fig. 4B). SEM images of complexed NCs show numerous uniform spherically shaped particles below $1000 \mathrm{~nm}$ with a lipid coat onto the surface (Fig. 4C). Fig. 4D shows images of complexed NCs (Batch F1) of reduced size after being dispersed in water and then air dried. Fig. 4E shows aggregated NCs before complexation and after microscopical examination. These observations reveal a distinct difference in surface morphology of pure GLP and NCs. 

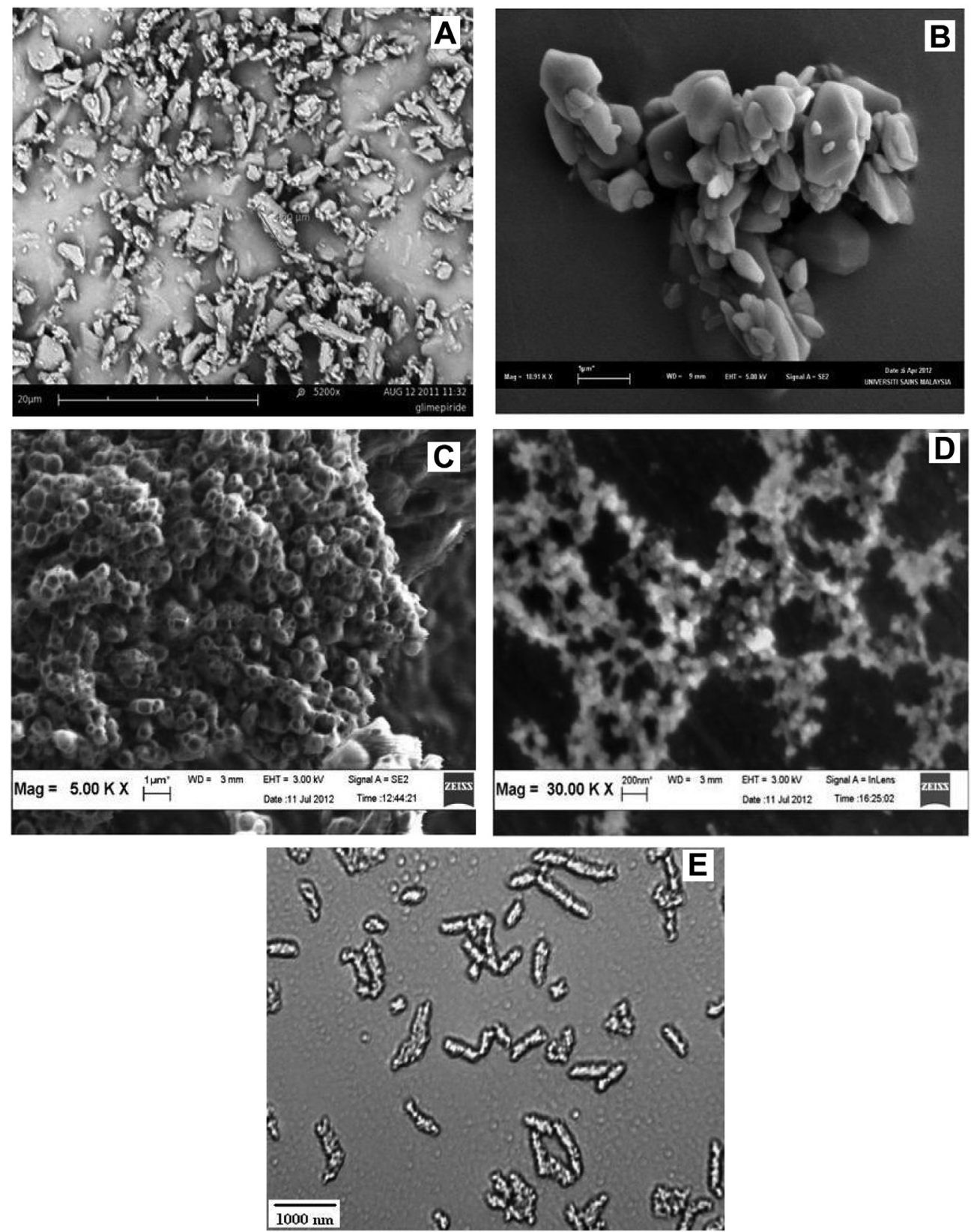

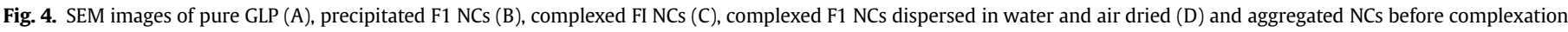
and after microscopical examination (E).

\subsection{Stability testing}

The stability data of optimized batch (F1) is given in Table 3. No significant change in particle size was observed during the storage period. FTIR spectra of optimized formulation (batch F1) were found to possess the characteristic peaks of pure GLP at specific positions and are given in Fig. 3B (GLP F1 SO).

\section{Discussion}

GLP NCs were formulated by precipitation technique and complexed using P90G (lipid). Particle and solid-state characterization studies were performed on NCs at various stages of its development and the factors were optimized. The effect of PEG 20000 and P90G on particle size reduction, crystallinity and stability of NCs were also assessed.

The average particle size was found to vary (30-1950 nm) with polymer content in precipitated NCs. This could be due to instabilization of particles as a result of crystal growth. The average particle size was found to be lower in complexed NCs, possibly due to

Table 3

Stability data of optimized NCs (Batch F1).

\begin{tabular}{lllll}
\hline Stability conditions & \multicolumn{4}{l}{ Observation (months) } \\
\cline { 2 - 5 } & 0 & 1 & 2 & 3 \\
\hline Room temperature & $240.4 \pm 0.027$ & $240.1 \pm 0.73$ & $241.5 \pm 0.085$ & $241.7 \pm 0.161$ \\
$40^{\circ} \mathrm{C}(\mathrm{RH}=75 \%)$ & $240.4 \pm 0.019$ & $240.8 \pm 0.17$ & $241.5 \pm 0.179$ & $242.7 \pm 0.158$ \\
\hline Values represent Z.avg $(\mathrm{d} \mathrm{nm}), \pm$ indicates SD $(n=3)$.
\end{tabular}


less aggregation of particles. ${ }^{19}$ The increase in PDI before and after complexation could be as a result of application of less energy during the process of milling. All the batches were polydisperse in nature after complexation. It can be inferred that batch F1 was found to be the optimum batch in terms of lowest particle size and stability, and a drug-polymer ratio of $1: 1$ was most suitable to develop stable NCs.

The average zeta values were found to increase after complexation. This is because of formation of a lipid coat on the surface of NCs. Lipid (P90G) imparts a negative charge, makes the drug particles to get dissociated and provides maximum stability to NCs. These findings also suggest that a repulsive force is associated with the molecules which reduce the particle agglomeration and it confirms the stability of the formulation. ${ }^{20}$

XRPD studies show no change in crystallinity in precipitated and complexed NCs. This was evident from relative intensity values ( $d$ value), as it decreased initially and later became constant. A shift in diffraction pattern was noticed at a longer end in $2 \theta$ scale, indicating the change in crystal size due to PEG $20000 .^{21}$ It was observed that all NCs exhibited a similar characteristic diffraction pattern as that of pure GLP at $2 \theta$ positions revealing the absence of interaction between drug and polymer.

DSC studies confirmed that crystallinity was maintained in all complexed NCs but with a slight degree of disorientation in their crystal structure. This could be due to the change in $\Delta H(\mathrm{~J} / \mathrm{g})$ values. The variation in peak and $\Delta H$ value of NCs supports the above suggestions.

The surface morphology of precipitated and complexed NCs was easily distinguishable by SEM analysis. The complexed NCs were spherical in nature and the particle size was below $1 \mu \mathrm{m}$. Dispersion of these NCs in water exhibited less particle aggregation due to the presence of lipids on the surface of NCs. Stability assessment on optimized formulation (Batch F1) by FTIR analysis and particle size measurement showed no change in characteristic peak and particle size. Thus the chemical identity of GLP was preserved in the samples and the formulation was stable during the period.

The particle analysis report and solid-state characterization studies reveals that in vitro stability of NCs were improved and the chemical identity of the drug was not altered during the process of complexation. This confirms that the formulation is stable and complexed NCs can be effectively used in design of various formulations. However, for assessing the complete safety and efficacy in drug delivery in vivo studies ought to be performed, as there is a possibility for the NCs to experience a change in surface properties in presence of body fluids. This could be assessed by performing permeability studies and by analyzing the biodistribution properties of NCs.

\section{Conclusion}

Particle analysis and solid-state characterization studies portray the drug properties, its stability, and compatibility of drug and excipients. These studies are essential at various stages of development of lipid nanocrystals, as it would assist in design of an uncomplicated formulation. These complexed NCs are promising carriers for development of various formulations in treatment of diabetes. To conclude, PEG 20000 is an excellent polymer for nanonization process and P90G could be effectively used for complexation as it can alter the surface properties, maintain crystallinity and improve in vitro stability.

\section{Conflicts of interest}

All authors have none to declare.

\section{Acknowledgments}

The authors would like to thank Faculty of Pharmacy and Faculty of Applied Sciences, AIMST University, Malaysia and SAIF-STIC, Cochin, India for their laboratory and instrumentation analysis support.

\section{References}

1. Jens UA, Rainer HM. Nanocrystal technology drug delivery and clinical applications. Int J Nanomed. 2008;3:295-299.

2. Huabing C, Chalermchai K, Xiangliang Y, Xueling C, Jinming G. Nanonization strategies for poorly water soluble drugs. Drug Discov Today. 2011;16:354-360.

3. Marcato PD, Duran N. New aspects of nanopharmaceutical delivery systems. J Nanosci Nanotech. 2008:8:2216-2229.

4. Rainer HM, Sven G, Cornelia MK. State of art nanocrystals - special features, production, nanotoxicity aspects and intracellular delivery. Eur J Pharm Biopharm. 2011;78:1-9.

5. Banu SZ, Nakissa S. Regulatory perspective on the importance of ADME assessment of nanoscale material containing drugs. Adv Drug Deliver Rev. 2009;6:422-427.

6. Mohanraj VJ, Chen Y. Nanoparticles - a review. Trop J Pharm Res. 2006;5:561573.

7. Tom A, Max Z. Formulation technologies to overcome poor drug like properties. Drug Discov Today. 2012;9:e71-e72.

8. Lai F, Sinico C, Ennas G, Marogiu F, Marogiu G, Fadda AM. Diclofenac nanosuspensions: influence of preparation procedure and crystal form on drug dissolution behaviour. Int J Pharm. 2009;373:124-132.

9. Faris NB, Müller RH. Nanocrystals of poorly soluble drugs for oral administration. Topics of PhD thesis and details on technologies, products, IP. New Drugs. 2002;2:20-21.

10. Jaime S, Antoine G, Rainer HM, Jan PM. Nanocrystals: comparison of the size reduction effectiveness of a novel combinative method with conventional topdown approaches. Eur J Pharm Biopharm. 2012;81:82-90.

11. Sandrine D, Lucie S, Jean LC. Physico-chemical parameters that govern nanoparticles fate also dictate rules for their molecular evolution. Adv Drug Deliver Rev. 2012;64:179-189.

12. Zeng N, Gao X, Hu Q et al. Lipid-based liquid crystalline nanoparticles as oral drug delivery vehicles for poorly water-soluble drugs: cellular interaction and in vivo absorption. Int I Nanomed. 2012:7:3703-3718.

13. Müller RH, Jacobs C, Kayser O. Nanosuspensions as particulate drug formulations in therapy, rationale for development and what we can expect for the future. Adv Drug Deliver Rev. 2001:47:3-19.

14. Shelesh J, Swarnalata S. Type 2 diabetes mellitus - Its global prevalence and therapeutic strategies. Diabetes Metab Syndr Clini Res Rev. 2010;4:48-56.

15. Vyas M, Galani VJ. In vivo and in vitro drug interactions study of glimepiride and atorvastatin and rosuvastatin. I Young Pharmacist. 2010;2:196-200.

16. Sajeev KB, Saraswathi R, Dilip C, Venkates K, Jha SK. Formulation and evaluation of controlled release glimepiride osmotic systems. Int J Pharm Res. 2011;3: 79-84.

17. Rhoban T. The efficacy and safety of glimepiride in the management of Type II diabetes in Muslim patients during Ramadan. Diabetes Care. 2005;28:421-422.

18. Baliar S, Biswal S, Sahoo J, Murthy PN. Physiochemical properties of glimepiride in solid dispersions with polyethylene glycol 20000. Int J Pharm Sci Nano. 2009;2:537-543.

19. Marie G, Angelica V, Robert G, Florence D. Nanoparticles for drug delivery: the need for precision in reporting particle size parameters. Eur J Pharm Biopharm. 2008;69:1-9.

20. Jane W, Sabine G, Heather O, Lakshmy N, Thomas G, Philip WG. Physicochemical stability of phospholipid-dispersed suspensions of crystalline itraconazole. Eur I Pharm Biopharm. 2008;69:1104-1113.

21. Anjan K, Mahapatra. Dissolution enhancement and physiochemical characterization of fenofibrate in solid dispersion with polyethylene glycol 40000 and 20000. Int J Pharm Sci Tech. 2010;4:21-31. 